\title{
Novel treatments for osteoporosis
}

\author{
Ernesto Canalis
}

See related articles,

Departments of Research and Medicine, Saint Francis Hospital and Medical Center, Hartford, Connecticut, USA; and Departments of Medicine and Orthopedics, The University of Connecticut School of Medicine, Farmington, Connecticut, USA

Address correspondence to: Ernesto Canalis, Department of Research, Saint Francis Hospital and Medical Center, 114 Woodland Street, Hartford, Connecticut 06105-1299, USA. Phone: (860) 714-4068; Fax: (860) 714-8053;

E-mail: ecanalis@stfranciscare.org.

Osteoporosis is a major health problem that affects 4 to 6 million women and 1 to 2 million men in the US, with an even larger number of individuals having osteopenia or decreased bone mineral density (BMD) (1). The most significant consequence of osteoporosis is osteoporotic fractures, which in 1995 resulted in health care expenditures in the US of $\$ 13.8$ billion (2). Consequently, the prevention and treatment of osteoporosis are of paramount importance. Currently, all therapeutic agents approved by the Food and Drug Administration for the treatment of osteoporosis are inhibitors of bone resorption. Although these agents are effective in stabilizing or increasing BMD and reducing the risk of fractures, their exact mechanism of action frequently is not known, and they do not increase bone formation. Alternative drug therapies that either block the function of the bone-resorbing osteoclasts or enhance the anabolic function of osteoblasts could prove beneficial to a number of patients with osteoporosis. A recent issue and, now, the current issue of the JCI feature studies on two promising new agents that operate by these complementary strategies and preserve bone mass to a significant extent in rat models of osteoporosis $(3,4)$. Figure 1 shows the effect of these agents on the balance between ongoing processes of bone formation and bone resorption, which together determine bone mass.

\section{Blocking bone matrix acidification} Vacuolar ATPases (V-ATPases) are multisubunit enzymes that transport protons across limiting membranes. V-ATPases are ubiquitously distributed among all cells and are evolutionarily conserved. These proton pumps are present at the plasma membrane of the kidney tubule intercolated cell and in the ruffled border of the osteoclast, where they mediate the acidification of the extracellular environment (5) and, thus, help to solubilize bone mineral. $V$-type proton pumps have two functional domains: a peripherally associated cytoplasmic catalytic section composed of four subunits, and a proton channel composed of three subunits (6). One of these latter components, the 116$\mathrm{kDa}$ subunit of the proton channel, is expressed exclusively in osteoclasts (6) and confers unique functional and pharmacological properties to the osteoclastic proton pump (5).

The functional relevance of the 116$\mathrm{kDa}$ subunit was confirmed by the severe osteopetrotic phenotype of mice lacking exons 2 to 5 of Apt6i, the gene encoding this subunit (7). In addition, these mice display shortened limbs, lack incisors and molars, and have trabeculae that obliterate the marrow space. The osteopetrosis observed in the Atp6inull mutants is not due to lack of osteoclast formation, since the number and appearance of these cells are normal, but rather due to a lack of osteoclastic function. These animals have a normally functional renal proton pump, confirming that the $116-\mathrm{kDa}$ subunit is specific to the osteoclast and that the osteoclast proton pump is structurally and functionally distinct from other proton pumps. The findings in the knock-out mouse are paralleled by those occurring in a spontaneous form of murine osteopetrosis, in which animals carry a 1579-bp deletion that removes the translation start site from Atp6i. A form of human autosomal recessive osteopetrosis that is lethal in the first 10 years of life is localized to chromosome 11q13, where the human orthologue of this gene would be expected to map, based on conserved synteny. This disease is similar in several respects to the phenotype of the mutant mice, suggesting that mutations in the human ATP6I indeed underlie the disease (8).

These genetic data suggested that reduced function of the $116 \mathrm{kDa}$ subunit might ameliorate osteoporosis, an insight that led to the development of a new class of inhibitors of osteoclastic function, acting selectively on the proton pump. (2Z,4E)-5-(5,6-dichloro-2indonyl)-2-methoxy- $N$-(1,2,2,6,6-pentamethylpiperidin-4-yl)-2,4-pentadiena mide (SB 242784), a compound derived from the potent V-ATPase inhibitor bafilomycin $\mathrm{A}_{1}$, inhibits the osteoclastic proton pump and bone resorption in vitro (9). Although it inhibits the activity of nonosteoclastic proton pumps, it appears to have reasonable selectivity for the osteoclastic enzyme. In this issue of the JCI, Visentin et al. characterize in detail the in vivo actions of SB 242784 (3), showing that the compound inhibited osteoclast ATPase activity in a dose-related manner from 0.01 to 100 $\mu \mathrm{M}$, but that it inhibited liver and kidney ATPases only at $100 \mu \mathrm{M}$.

SB 242784 inhibited retinoid-induced hypercalcemia in thyroparathyroidectomized rats, and its administration for 6 months prevented the loss of femoral and vertebral BMD in ovariectomized rats by decreasing bone remodeling (3). It is important to note that SB 242784 was as effective as, but not more effective than, estradiol in ovariectomized rats. SB 242784 and estrogens might have an additive effect, as has been shown for other antiresorptive agents, but this possibility was not tested in the present study. Histomorphometric analysis confirmed that SB 242784, like estradiol, normalized trabecular number and prevented the bone resorption induced by ovariectomy. SB 242784 did not have significant renal effects in control and acid-loaded animals. Although the compound is of interest because it modifies a specific function of the osteoclast, clinical trials might or might not demonstrate that it has significant advantages over other inhibitors of bone resorption for the treatment of osteoporosis. 


\section{Figure 1}

Two novel approaches to increasing bone mass. Bone mass reflects the relative rates of bone formation, mediated by osteoblasts, and of bone resorption, mediated by osteoclasts. Parathyroid hormone (PTH) has complex effects on bone mass, since it stimulates both of these processes, but treatment with estrogen, bisphoshponates, or other agents specifically blocks its effects on bone resorption. PTH promotes bone formation by several mechanisms, enhancing the proliferation of cells of the osteoblastic lineage, and promoting osteoblastic function. Some of these anabolic effects of PTH are mediated by IGF I. PTH secretion from the parathyroid gland is suppressed by signaling through the calcium receptor (CaR), which senses extracellular calcium levels. The new drug NPS 2143 acts by reducing the sensitivity of the calcium receptor to extracellular calcium, thereby enhancing PTH secretion and promoting osteoblast function. The drug SB 242784 acts by a complementary strategy, specifically inhibiting the vacuolar ATPase and thereby blocking osteoclast-mediated acidification of the bone matrix, which is required for bone resorption.

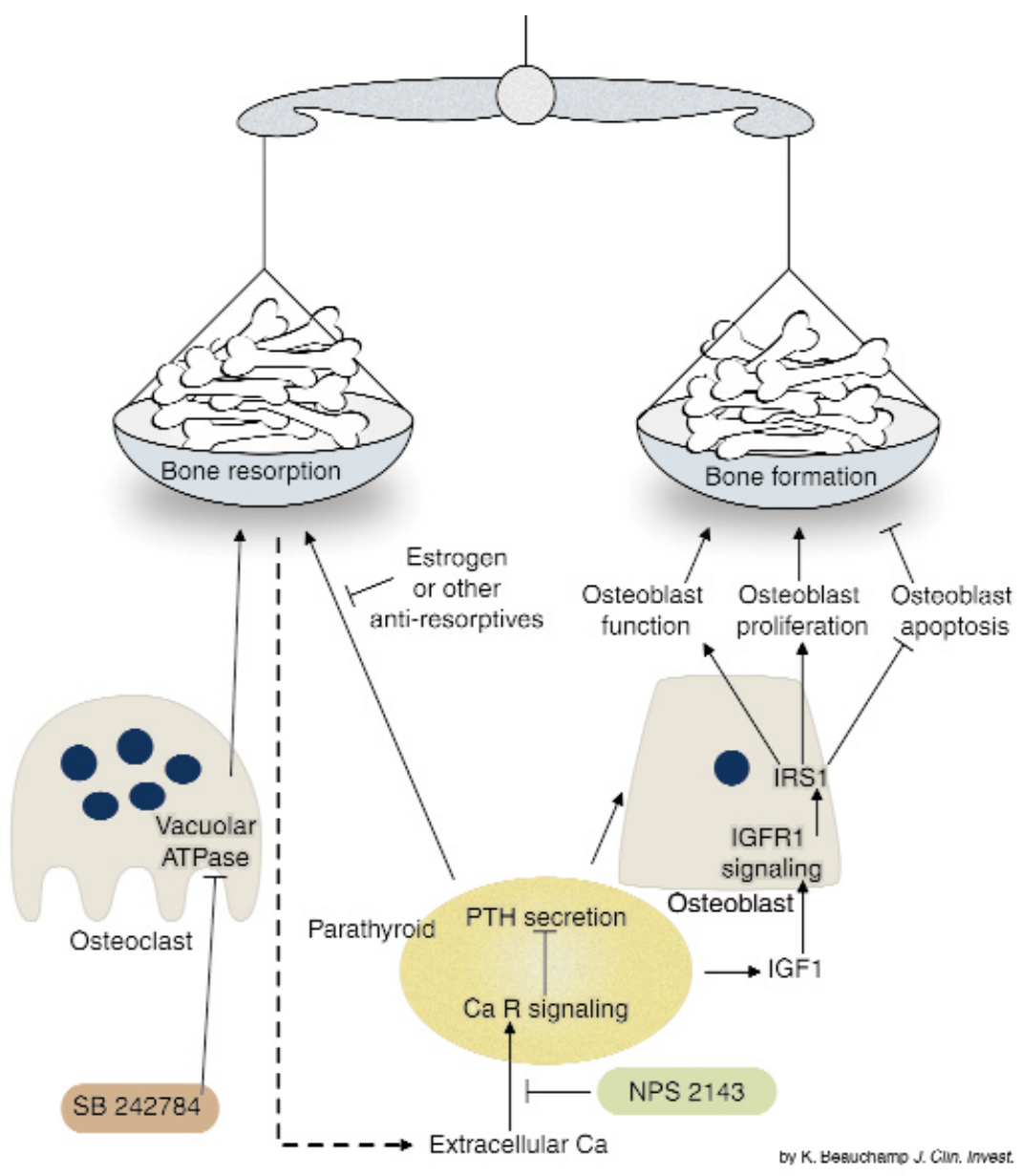

roidism (12). Similarly, heterozygous null mice lacking the calcium receptor develop hypercalcemia and hypocalciuria, and homozygous animals present with severe hyperparathyroidism and die prematurely. Activating mutations, conversely, result in hypocalcemia (13).

The discovery of the calcium receptor and its related clinical disorders led to the subsequent development of drugs that could modulate its activity: "calcimimetic" agents, which sensitize the receptor to calcium, and "calcilytic" agents, which have the opposite effect. By making the parathyroid cells more sensitive to calcium, calcimimetic agents reduce the levels of PTH. One such drug, the phenylalkylamine R-568, has been used successfully for treating hyperparathyroidism, and it decreases parathyroid hyperplasia in an experimental model of uremia (14-16).

In a recent issue of the JCI, Gowen et al. described an orally administered calcilytic agent (NPS 2143) that causes an acute increase in serum PTH levels, which are sustained for at least 8 hours (4). This effect is in contrast to the more transient elevation that follows the subcutaneous administration of PTH. Gowen et al. reported the expected increase in BMD of the tibiae of ovariectomized rats, to preovariectomized levels, following intermittent treatment with parenteral PTH. Surprisingly, NPS 2143 did not increase BMD, apparently because sustained high levels of PTH also induced a marked increase in bone resorption. Histomorphometric analysis revealed that NPS 2143 increased parameters of both bone formation and bone resorption, as well as increasing bone turnover. Nevertheless, coadministration of the calcilytic agent with estrogens to ovariectomized rats resulted in a greater increase in BMD and bone formation rate than estradiol alone, suggesting that blocking the resorptive effect of PTH with estrogens can uncover its anabolic actions. Whether antiresorptive agents obliterate the anabolic effect of PTH has been a controversial point, but most studies show no impact on the anabolic action of the hormone. Consequently, it is not surprising that the calcilytic agent was 
effective in the presence of estradiol. The agent had no direct effects on osteoblasts or osteoclasts and did not cause parathyroid hyperplasia.

Although PTH alone prevents the bone loss of the spine secondary to hypogonadism, recent trials in women with postmenopausal osteoporosis, like the study reported in the JCI (4), have examined the effect of PTH in conjunction with estrogens. In one study, daily treatment with PTH and estrogens for 3 years caused an increase of $13 \%$ in BMD of the spine and of $2.7 \%$ in BMD of the hip (17). In a second study, the effects of PTH and estrogens were more pronounced, increasing BMD of the lumbar spine and femoral neck by $29 \%$ and $11 \%$, respectively, after 2 years (18). These investigations document that PTH and estrogens cause a large increase in BMD. A possible use of a calcilytic agent is in the treatment of glucocorticoid-induced osteoporosis, since initial studies indicate that PTH is effective in this condition. Although glucocorticoids increase bone resorption, their inhibitory effects on bone formation play a central role in the development of osteoporosis, due to direct actions of glucocorticoids on osteoblastic function and number and to an inhibition of IGF I synthesis (19). This effect is reversed by PTH in vitro and may explain its therapeutic effectiveness in glucocorticoid-induced osteoporosis. In a group of postmenopausal women with glucocorticoid-induced osteoporosis, supplementing estrogen regimens with daily PTH increased BMD of the spine and hip by $9.8 \%$ and $2 \%$, respectively, within a year (20). In contrast, BMD remained unchanged in patients treated with estrogens alone.

The mechanisms responsible for the anabolic effect of the calcilytic agent in bone have not been reported, but it is likely that they are analogous to those of PTH. This hormone has mitogenic properties for cells of the osteoblastic lineage and increases the synthesis of IGF I by osteoblasts, resulting in an increase in bone collagen synthesis and bone formation (21). In addition, PTH increases skeletal levels of IGF I and TGF- $\beta 1$ in experimental animals, and these effects correlate with an increase in BMD (22). The importance of IGF I in the maintenance of bone mass was recently substantiated in mice lacking the insulin receptor substrate- 1 , which is essential for insulin and IGF I signaling (23). These mice develop lowturnover osteoporosis secondary to decreased bone formation, as a consequence of decreased osteoblastogenesis and osteoclastogenesis. Recently, PTH was reported to increase bone mass in normal and osteoblast-deficient mice by decreasing osteoblastic apoptosis, a mechanism that could increase the number of bone producing cells (24). The possibility of administering an oral agent that can enhance PTH secretion offers an exciting novel therapeutic avenue for the treatment of osteoporosis, but appropriate clinical trials will be needed to demonstrate its effectiveness in this disease.

\section{Acknowledgments}

The author thanks Karen V. Berrelli for secretarial assistance.

1. Looker, A.C., et al. 1997. Prevalence of low femoral bone density in older U.S. adults from NHANES III. J. Bone Miner. Res. 12:1761-1768.

2. Ray, N.F., Chan, J.K., Thamer, M., and Melton, L.J., III. 1997. Medical expenditures for the treatment of osteoporotic fractures in the United States in 1995: report from the National Osteoporosis Foundation. J. Bone Miner. Res. 12:24-35.

3. Visentin, L., et al. 2000. A selective inhibitor of the osteoclast $\mathrm{V}-\mathrm{H}^{+}$-ATPase prevents bone loss in both thyroparathyroidectomized and ovariectomized rats. J. Clin. Invest. 106:309-318.

4. Gowen, M., et al. 2000. Antagonizing the parathyroid calcium receptor stimulates parathyroid hormone secretion and bone formation in osteopenic rats. J. Clin. Invest. 105:1595-1604.

5. Chatterjee, D., et al. 1992. Sensitivity to vanadate and isoforms of subunits $\mathrm{A}$ and $\mathrm{B}$ distinguish the osteoclast proton pump from other vacuolar $\mathrm{H}^{+}$ ATPases. Proc. Natl. Acad. Sci. USA. 89:6257-6261.

6. Li, Y.-P., Chen, W., and Stashenko, P. 1996. Molecular cloning and characterization of a putative novel human osteoclast-specific 116-kDa vacuolar proton pump subunit. Biochem. Biophys. Res. Commun. 218:813-821.

7. Li, Y.-P., Chen, W., Liang, Y., Li, E., and Stashenko, P. 1999. Atp6i-deficient mice exhibit severe osteopetrosis due to loss of osteoclast-mediated extracellular acidification. Nat. Genet. 23:447-452.
8. Scimeca, J.-C., et al. 2000. The gene encoding the mouse homologue of the human osteoclast-specific 116-kDa V-ATPase subunit bears a deletion in osteosclerotic (oc/oc) mutants. Bone. 26:207-213.

9. Nadler, G., et al. 1998. (2Z,4E)-5-(5,6-dichloro-2indolyl)-2-methoxy-n-(1,2,2,6,6-pentamethylpiperidin-4YL)-2,4-pentadienamide, a novel, potent and selective inhibitor of the osteoclast V-ATPase. Bioorg. Med. Chem. Lett. 8:3621-3626.

10. Brown, E.M., et al. 1993. Cloning and characterization of an extracellular $\mathrm{Ca}^{2+}$-sensing receptor from bovine parathyroid. Nature. 366:575-580.

11. Brown, E.M., Pollak, M., and Hebert, S.C. 1998. The extracellular calcium-sensing receptor: its role in health and disease. Annu. Rev. Med. 49:15-29.

12. Pollak, M.R., et al. 1993. Mutations in the human $\mathrm{Ca}^{2+}$-sensing receptor gene cause familial hypocalciuric hypercalcemia and neonatal severe hyperparathyroidism. Cell. 75:1297-1303.

13. Watanabe, T., et al. 1998. Familial hypoparathyroidism: identification of a novel gain of function mutation in transmembrane domain 5 of the calcium-sensing receptor. J. Clin. Endocrinol. Metab. 83:2497-2502.

14. Nemeth, E.F., et al. 1998. Calcimimetics with potent and selective activity on the parathyroid calcium receptor. Proc. Natl. Acad. Sci. USA. 95:4040-4045.

15. Silverberg, S.J., et al. 1997. Short-term inhibition of parathyroid hormone secretion by a calciumreceptor agonist in patients with primary hyperparathyroidism. N. Engl. J. Med. 337:1506-1510.

16. Wada, M., et al. 1997. The calcimimetic compound NPS R-568 suppresses parathyroid cell proliferation in rats with renal insufficiency. $J$. Clin. Invest. 100:2977-2983.

17. Lindsay, R., et al. 1997. Randomised controlled study of effect of parathyroid hormone on vertebral-bone mass and fracture incidence among postmenopausal women on oestrogen with osteoporosis. Lancet. 350:550-555.

18. Cann, C.E., Roe, E.B., Sanchez, S.D., and Arnaud, C.D. 1999. PTH effects in the femur: envelopespecific responses by 3DQCT in postmenopausal women. J. Bone Miner. Res. 14:S137. (Abstr.)

19. Canalis, E. 1996. Clinical Review 83: Mechanisms of glucocorticoid action in bone: implications to glucocorticoid-induced osteoporosis. J. Clin. Endocrinol. Metab. 81:3441-3447.

20. Lane, N.E., et al. 1998. Parathyroid hormone treatment can reverse corticosteroid-induced osteoporosis. Results of a randomized controlled clinical trial. J. Clin. Invest. 102:1627-1633.

21. Canalis, E., Centrella, M., Burch, W., and McCarthy, T.L. 1989. Insulin-like growth factor I mediates selective anabolic effects of parathyroid hormone in bone cultures. J. Clin. Invest. 83:60-65.

22. Pfeilschifter, J., et al. 1995. Parathyroid hormone increases the concentration of insulin-like growth factor-I and transforming growth factor beta 1 in rat bone. J. Clin. Invest. 96:767-774.

23. Ogata, N., et al. 2000. Insulin receptor substrate1 in osteoblast is indispensable for maintaining bone turnover. J. Clin. Invest. 105:935-943.

24. Jilka, R.L., et al. 1999. Increased bone formation by prevention of osteoblast apoptosis with parathyroid hormone. J. Clin. Invest. 104:439-446. 